NACIONAL

\title{
El derecho al nombre social en las atenciones de salud respecto de pacientes transgéneros menores de edad: El caso Araya Astudillo con Clínica Alemana
}

\author{
The right to a social name in health care with respect of minor transgender patients: \\ The case Araya Astudillo v. Clínica Alemana
}

\author{
Pablo Eterovic Barreda \\ Universidad de Chile
}

\begin{abstract}
RESUMEN A cinco años de la entrada en vigor de la Ley 20.584, que regula los derechos y deberes en relación con acciones vinculadas a la atención en salud, las personas transgéneros siguen viendo vulnerados sus derechos en esta materia, en específico el derecho a recibir un trato digno y ser llamados por su nombre, toda vez que no se respeta su nombre social ni éste ha sido incorporado en su ficha clínica. El presente artículo tiene por objeto establecer el marco jurídico vigente respecto de las personas transgénero y el derecho que tienen a que su nombre social sea considerado en materia sanitaria, incluso en el sector privado, aun a falta de norma expresa, con un comentario en específico sobre una sentencia de la Corte de Apelaciones de Santiago dictada el 6 de octubre de 2016 y ratificada luego por la Corte Suprema el 14 de noviembre del mismo año, que establece esta obligación respecto de un menor de edad, sin que dicho derecho esté condicionado a la rectificación de partida de nacimiento.
\end{abstract}

PALABRAS CLAVE Derechos de los pacientes, derecho al nombre social, ficha clínica, personas transgéneros, interés superior del niño.

ABSTRACT Almost five years after the enforcement of Law 20,584, which regulates the rights and duties in relation to health care, transgender people continue to see their rights in this matter violated, specifically the right to receive a decent treatment and be called by their name, as long as their social name is not respected nor has it been incorporated into their medical record. This article aims to establish the legal framework currently in force under which transgender people have the right to have their social name considered in health matters at a private clinic, even in the absence of an express rule, commenting specifically on a judgment of the Court of Appeals of Santiago issued on October 6, 2016 and subsequently ratified by the Supreme Court on November 14, 
under which this obligation is established with respect to a minor, right that cannot be conditioned to the legal procedure to rectify the birth certificate.

KEYWORDS Health care rights, right to a social name, medical records, transgender people, best interests of the child.

\section{Introducción}

Si bien el debate sobre los derechos de las personas transgéneros - es decir, las personas cuya identidad de género ${ }^{1}$ difiere en definitiva del sexo y género que se la ha asignado documentalmente al momento de su inscripción- está instalado en el mundo social y político, no deja de ser cierto que la «reivindicación» de sus derechos parece encontrarse en un estado de eterno aletargamiento. Lo anterior en cuanto la supuesta solución transversal a sus problemas lleva años de discusión sin poder concretarse en un cuerpo normativo acorde a los estándares internacionales. Hablamos del proyecto de ley que reconoce y da protección al derecho a la identidad de género presentado el 7 de mayo del 2013 (Boletín 8924-07), de actual discusión en el Senado.

Aunque el panorama a nivel legislativo no parece del todo prometedor, lo cierto es que las personas transgéneros, y en específico los niños, niñas y adolescentes, han logrado algunas victorias en ámbitos específicos de la vida social. Esos triunfos aislados se manifiestan ordenando que se les reconozca por su nombre social, lo que ha sucedido recientemente en materia de educación ${ }^{2} y$, como veremos en este trabajo, en materia de salud respecto la Red Nacional de Servicios de Salud. El nombre, de esta manera, se vuelve un verdadero atributo de la personalidad reconociéndose parte fundamental en la construcción de la identidad de una persona.

A pesar de ser esperanzadores, estos logros deben ser dimensionados en su justa medida. A falta de consagración legal vinculante de forma general, la tónica es que las personas transgéneros vean vulnerados sus derechos más de lo que nos gustaría

1. De acuerdo a los principios sobre la aplicación internacional de los derechos humanos respecto a la orientación sexual y la identidad de género, también conocidos como Principios de Yogyakarta, se refiere a la vivencia interna e individual del género tal como cada persona la siente profundamente, la cual podría corresponder o no con el sexo asignado al momento del nacimiento, incluyendo la vivencia personal del cuerpo (que podría involucrar la modificación de la apariencia o la función corporal a través de medios médicos, quirúrgicos o de otra índole, siempre que la misma sea libremente escogida) y otras expresiones de género, como la vestimenta, el modo de hablar y los modales.

2. El 27 de abril de 2017 la Superintendencia de Educación emitió la Circular o768 que regula los derechos de niñas, niños y estudiantes trans en el ámbito de educación. Su contenido, que resulta aplicable a todos los establecimientos educacionales sin excepción, establece dentro de las medidas básicas de apoyo el uso del nombre social en todos los espacios educativos y, en la medida de lo posible, los documentos oficiales, sin que ello constituya infracción a las disposiciones vigentes. 
asumir. En específico, la situación se agrava cuando concurren, además, otras circunstancias que en nuestro país se vuelven sinónimo de discriminación o parecen justificar la adopción de una postura paternalista más allá de todo criterio razonable, como, por ejemplo, cuando un niño, niña o adolescente transgénero requiere atención de salud, circunstancia en la que vive vulneraciones a sus derechos como minoría sexual, menor de edad y paciente. Su situación podría verse agravada en cuanto se discute si los niños, niñas y adolescentes podrán ser titulares de los derechos que el proyecto de ley consagraría ${ }^{3}$.

El presente trabajo busca demostrar, a propósito de un pronunciamiento de nuestros Tribunales Superiores de Justicia, que en el sector privado y respecto del trato conforme a su nombre social y el registro de él en la documentación clínica, basta la normativa vigente, en la medida en que se interprete armónicamente la normativa internacional (principalmente la Convención de Naciones Unidas Sobre los Derechos del Niño), los principios que subyacen a la Ley 20.584 y se promueva la adopción en los privados de los estándares por los cuales hoy en día se rige el sector público.

\section{El derecho a ser tratado por el nombre y a una ficha clínica en el derecho chileno}

Tras años de discusión, fue publicada el 24 de abril de 2012 en el Diario Oficial la Ley 20.584. Dicho texto legal se encuadra dentro de lo que se conoce como el tercer momento en la visión histórica de los derechos de los pacientes, con la formalización de sus derechos en cuerpos normativos específicos, basados ellos principalmente en el respeto al principio de autonomía del paciente (Terribas, 2012: 181). En cuanto al ordenamiento jurídico nacional, la Ley 20.584 vino a ser el cierre a la reforma del sistema de salud, teniendo como pilares fundamentales la Ley 19.966 de Régimen de garantías en salud; la nueva regulación de Isapres (Ley 20.015) y la nueva ley de autoridad sanitaria y sistemas de gestión (Ley 19.937).

De acuerdo con el Mensaje de la Presidencia de la República, la Ley 20.584 buscaba la aplicación de los derechos fundamentales a situaciones concretas que se producen en el escenario salud, se trate de prestadores públicos o privados. El proyecto estaba motivado por los principios de dignidad en materia sanitaria, el respeto al ejercicio de decisiones autónomas, el derecho de las personas a decidir informada-

3. En efecto, durante la tramitación del proyecto en la Comisión de Derechos Humanos, Nacionalidad y Ciudadanía del Senado, se ha discutido fuertemente la posibilidad de que los menores de edad puedan acogerse a este procedimiento, y la forma de dicho procedimiento de reconocimiento de la identidad de género (administrativa o judicial, esta última de manera contenciosa o no). A junio de 2017, el proyecto de ley que ha sido enviado desde la Comisión al Senado para su discusión en sala permite al oficial del Registro Civil declarar inadmisible la solicitud del menor de edad, pues restringe el procedimiento a quienes han alcanzado la mayoría. 
mente, el respeto de los menores de edad en las decisiones que involucran su cuerpo y salud incorporando ideas de capacidad progresiva, etcétera ${ }^{4}$.

\section{Derecho a ser tratado por el nombre}

\section{Consagración del derecho}

El título 2 de la Ley 20.584 está dedicado a los derechos que las personas tienen en materia de salud, al establecer como garantía general el derecho a un trato digno. Así, en el artículo 5 se establece que las personas tienen derecho a recibir un trato digno y respetuoso en todo momento y en cualquier circunstancia. En consecuencia, los prestadores deberán velar por que se adopten actitudes que se ajusten a las normas de cortesía y amabilidad generalmente aceptadas, $y$ «por que las personas atendidas sean tratadas y llamadas por su nombre».

\section{El nombre bajo el contexto de la Ley 20.584}

Si bien el derecho al nombre está establecido de forma expresa en la Ley 20.584, su contenido no es claro, pues establece un derecho a ser tratado por él, pero no dice qué entiende por el mismo. La respuesta a esta pregunta tampoco viene dada por la historia de la Ley (este derecho siguió su tramitación sin comentarios ni modificaciones sustanciales).

\section{Circular 21 de la Subsecretaría de Redes Asistenciales del Ministerio de Salud, de 14 de junio de 2012}

Luego de una infructuosa primera instrucción a finales del año 2011, que quedó como una simple declaración de intenciones sujeta al «debido criterio» del funcionario e institución frente a las situaciones que se le fueran presentado 5 , la Circular 21 estableció de forma obligatoria que la identificación verbal de los pacientes transgéneros sea siempre mediante el nombre social, mientras que la documentación clínica debe señalar tanto el nombre legal como social, junto con ciertos protocolos al momento de la anamnesis, dentro de otras materias. De esta manera, la autoridad solventaba los dos primeros estadios que, en materia de salud, reclaman los transgéneros: un trato

\footnotetext{
4. Mensaje 223-354 de la Presidenta de la República, con el que inicia un proyecto de ley que regula los derechos y deberes que las personas tienen en relación con acciones vinculadas a su atención en salud, del 26 de julio de 2006. Historia de la Ley 20.584, página 10, disponible en http://bit.ly/2u8Vd41.

5. Ver para tales efectos la Circular 34, del 13 de septiembre de 2011, que instruye sobre la atención que debe brindarse a las personas transexuales y transgéneros, con lo que fortaleció las estrategias del Plan Hospital Amigo a personas de diversidad sexual, y el caso Michel Riquelme Norambuena con Ministerio de Salud, Consejo para la Transparencia, rol C1421-11.
} 
conforme a su identidad de género y la rectificación de la documentación conforme a dicha identidad.

¿Pero qué hacemos respecto de los pacientes de centros particulares? La Circular 21 establece ciertas instrucciones en virtud de la dignidad de personas que no serían exigibles a los establecimientos particulares. Por otro lado, el derecho al trato digno es reclamable en todo momento y cualquier circunstancia. ¿Vincula ello a las clínicas privadas? ¿Tienen los pacientes transgénero ciertos derechos a los ojos de un centro privado y otros a los ojos de un centro público?

\section{El derecho a una ficha clínica}

\section{Concepto de ficha clínica}

Si bien la Ley 20.584 otorga una definición legal de ficha clínica, creemos conveniente construir un concepto de ficha clínica que haga confluir los intereses jurídicamente relevantes al respecto: paciente, médicos o profesionales y administración. En función de lo anterior, la ficha clínica es el documento escrito, sin importar su soporte, desarrollado obligatoriamente por el facultativo, que emana de la relación comunicacional médico-paciente, en el que constan interpretaciones subjetivas del médico y el conjunto de análisis objetivo de exámenes, con el fin de establecer, a lo largo de un proceso asistencial, los distintos estados de evolución del paciente, su ingreso, tratamiento, pronóstico y estado hasta el alta, garantizando el ejercicio de la autonomía del paciente a través de la toma de decisiones informada y el correcto ejercicio de la profesión.

\section{Rol de la ficha clínica en el acto médico}

En la actualidad se vive un verdadero cambio de paradigma en la atención de los pacientes, situación que influye en la forma en que entendemos la ficha clínica como instrumento, en virtud de los avances en la bioética desde la irrupción del principialismo en la praxis médica. Hablamos de un cambio de "paradigma» y no simplemente normativo, pues se trata de un nuevo enfoque que, a falta de cambios legales, busca influir en la forma de practicar la salud en la actualidad proyectándose en modificaciones legislativas futuras, pero también influyendo en el ejercicio de la medicina, lo que abarca etapas formativas, de educación y de información, entre muchas otras.

La posición que podríamos denominar clásica o paternalista entiende la ficha clínica como una herramienta dedicada, exclusivamente, a facilitar la asistencia o tratamiento al paciente como herramienta del médico ${ }^{6}$. Sería una prolongación o mezcla

6. Para Ruiz Orejuela, «el fin principal de la historia clínica es facilitar la asistencia al ciudadano [...] por los sanitarios que le atienden. Esta finalidad es la razón de ser de la historia clínica» (Ruiz Orejuela, 2004: 131; el énfasis es nuestro). 
entre la memoria y el criterio del doctor, pensada en su exclusiva utilidad7. Así, el médico particular o su equipo de salud se vuelven los únicos destinatarios de ella. Este modelo, que se define como «modelo de eficacia terapéutica», promueve que la ficha clínica sea una herramienta en que esté toda la información necesaria y útil para el proceso asistencial del paciente, pero ello en beneficio y desde la perspectiva del médico ${ }^{8}$.

La posición moderna, en cambio, entiende la ficha clínica como un instrumento consagrado principalmente en beneficio de la autonomía del paciente. Este nuevo enfoque emana, para Martínez Aguado, de la dignidad moral y la condición de ciudadano del paciente (Fernández Hierro, 2002: 77). Lamentablemente, la Ley 20.584 terminó siendo un texto legal bastante esquizofrénico, en el que si bien se adhiere a la autonomía como principio general, lo cierto es que en determinados escenarios el principio ha sido directamente vulnerado".

Es en virtud de la autonomía de las personas y el respeto a su dignidad, que nos permitimos asegurar que una ficha clínica debe incorporar siempre el nombre social. Si un paciente tiene la libertad para consentir o no con determinado tratamiento, lo anterior a partir de la información que reciba y que se encuentre incorporada en su ficha clínica, con más razón puede regular el contenido no sanitario de ella. Un paciente con mayor sentido de pertenencia de su historial médico es un paciente más informado y, en consecuencia, un paciente más autónomo.

\section{El nombre como contenido de la ficha clínica}

Por expreso mandato de la Ley 20.584, el Ministerio de Salud se vio en la obligación de dictar un reglamento que complementase las disposiciones de la Ley 20.584 Lo anterior se realizó mediante la aprobación del Reglamento 41 sobre Fichas Clínicas, publicado en el Diario Oficial el 15 de diciembre de 2012.

En cuanto al contenido de la ficha clínica, el Reglamento señala en su artículo 6 ciertas condiciones mínimas sobre identificación actualizada del paciente, entre las que se encuentra el nombre completo. Qué se entiende por ello, no lo señala. El Ministerio de Salud tampoco ha sido capaz de aclararlo ${ }^{10}$. Con todo, en específico, respecto

7. Utilidad que no debe, necesariamente, entenderse en un sentido sanitario estricto, sino en uno amplio, pues cumple además roles preventivos y rehabilitadores (Fernández Hierro, 2002: 6).

8. Otra perspectiva del paso de un modelo paternalista a uno del «consentimiento informado» en Noriega Potocnjak (2011: 14).

9. El mensaje original señalaba que el proyecto buscaba respetar la dignidad de las personas, pero no como una mera afirmación declarativa o una bien fundada pretensión programática. Al contrario, «ésta se traducía en formulaciones y hechos concretos, especialmente en el respeto por su libertad». Mensaje de Ley 20.584, página 6.

10. Consultada al respecto, la ministra de Salud señaló que «no existen antecedentes que consten en 
de los «registros en ficha clínica», la Circular 21 ya señalada instruyó que todos los registros derivados de la atención de salud deben contemplar, en primer lugar, el nombre legal de la persona (consignado en su cédula de identidad) y, en segundo lugar, el nombre social con el cual dicha persona se identifica. Aunque dicha norma no sería vinculante en principio a los prestadores particulares, su influencia como principio inspirador no puede ser negada.

\section{Rol del nombre en la ficha clínica}

El único instrumento válido que permite vincular a una persona con una ficha clínica específica es el rol único nacional (RUN). No cumple este rol el nombre de la persona. Es respecto del RUN que el centro asistencial debe otorgar un número interno, en especial por su invariabilidad. Esto porque en el procedimiento de rectificación de partida de nacimiento se mantiene invariable el RUN.

En este sentido, el nombre viene a formar parte de lo que denominamos elemento de «identificación» del paciente, algo muy distinto de la «identidad». Este segundo concepto, que apunta a cualidades personales relevantes para el servicio de salud, se construye principalmente sobre la base de los elementos de anamnesis y diagnóstico (Ghersi y Weingarten, 2005: 51). Definida la identidad del paciente y vinculada ella a un RUN, es claro que un cambio del nombre registrado en la ficha clínica no afecta en ningún sentido la calidad de atención que recibe el paciente ni puede arriesgar su tratamiento. Por el contrario, es especialmente importante en el caso de personas cuyo nombre legal no es el que socialmente quiere utilizar, logrando que el paciente sea reconocido de acuerdo con su identidad de género.

\section{Rectificación de la ficha clínica y principios}

Se vuelve obligatorio incorporar el nombre social en la ficha clínica en virtud de los siguientes principios que la rigen:

- El principio de tutela de derechos del paciente, que busca el respeto no sólo de aquellos derechos de índole de la salud, sino todos aquellos derechos fundamentales del paciente, como la dignidad, la honra y la igualdad, entre otros.

- El principio de veracidad, el cual «se proyecta doblemente: por un lado, es requisito inexcusable de cualquier dato e información que se incluya en la his-

\footnotetext{
actas de comisiones, expedientes u otras actas administrativas en las cuales consulte el trabajo del Ministerio de Salud para la confección del reglamento de fichas clínicas, lo anterior debido a que se trabajó sobre la base de la misma Ley 20.584 y la Ley 19.628 sobre protección a la vida privada y datos de carácter personal». En caso Pablo Eterovic Barreda con Ministerio de Salud, Consejo para la Transparencia, rol C591-15. La respuesta específica en Ord. A/102 número 1407, del 22 de mayo de 2015.
} 
toria clínica; de otra parte, el conjunto de la información contenida en la historia clínica debe permitir un conocimiento cierto - veraz- de la situación del enfermo» (Rebollo Delgado y Gómez Sánchez, 2008: 285). El nombre social es el nombre con el cual la persona es y quiere ser conocida, pues va de la mano con su condición transgénero y construcción de su identidad.

\section{El caso Araya Astudillo con Clínica Alemana}

\section{Antecedentes}

El menor NN presentaba un diagnóstico de disforia de género, el cual había sido establecido incluso por el mismo centro asistencial contra quien se acciona. Identificada inicialmente como hombre, la menor había iniciado ya su tránsito social y los padres solicitaron al centro de salud que se le tratara como niña, de acuerdo a su identidad de género y respetando su nombre social. El centro asistencial señaló que lo anterior no era posible «toda vez que la información de los pacientes se asocia a su cédula de identidad, y para efectuar u otorgar cualquier atención, se requiere contar con la identificación y datos que ésta arroja». Concluyeron entonces que «mientras la identidad legal de una persona no es modificada legalmente, Clínica Alemana no puede cambiarla» ${ }^{11}$. A petición posterior de los padres, mantuvieron la negativa amparándose esta vez en el «marco legal referente a la verdadera identidad de un paciente» (Ley 4.808 sobre Registro Civil) y el mandato del Reglamento de señalar en la ficha clínica «la verdadera y real identidad de cada paciente». Actuar de distinta manera, sostuvieron, significaría una vulneración a los protocolos internos, toda vez que «la ficha clínica necesariamente debe ir asociada a la cédula de identidad de cada paciente, lo que importa su identificación precisa y real en todo aspecto, ello con el objeto de evitar cualquier confusión en torno a ello $»^{12}$. Los padres decidieron presentar el recurso en análisis, solicitando en específico que se ordene a la Clínica que incluya en la ficha clínica de la menor su identidad social y que asimismo se respete dicha identidad en todo ámbito de actuación.

A continuación, se analiza el fallo de primera instancia (sentencia del 6 de octubre de 2016, dictada por la Corte de Apelaciones de Santiago, rol 35.639-2016), que fuera ratificado luego por la Corte Suprema, con voto de minoría (sentencia del 14 de noviembre de 2016, rol 79.930-2016). Se debe tener presente, en todo caso, que durante la tramitación del recurso la causa voluntaria seguida ante el Séptimo Juzgado Civil de Santiago por rectificación de la partida de nombre había llegado a su fin, con lo que se acogió en definitiva la solicitud de los padres y se ordenó al Registro Civil la

11. Carta enviada por María Francisca Geisse Ovalle, jefa de Atención al Cliente, Clínica Alemana, 27 de enero de 2016.

12. Carta enviada por Eduardo Belmar García, abogado de Clínica Alemana, 8 de abril de 2016. 
rectificación correspondiente para dar cuenta del nuevo nombre y sexo ${ }^{13}$. Si bien el escenario que justificaba la negativa habría mutado durante la tramitación (tratar de acuerdo al nombre social incondicionalmente frente a tratar conforme al nombre social establecido en la sentencia hasta su total inscripción), los principios en uno y otro caso son los mismos y los Tribunales Superiores no desestimaron la oportunidad de pronunciarse.

\section{Análisis jurídico e importancia de la resolución}

\section{Violación por acción pero también omisión}

El primer elemento a considerar es que la clínica recurrida fue condenada por una acción (no tratar a la menor de acuerdo con su identidad social), pero también por una omisión (no incorporar su nombre social en su ficha clínica).

\section{Constante violación del derecho}

El recurso de protección acá analizado fue presentado con anterioridad en otras dos ocasiones, pero fue declarado inadmisible por encontrarse fuera de plazo, contado por la Corte de Apelaciones desde la carta de negativa recibida por los padres ${ }^{14}$, haciendo mención expresa en el último de los casos que «las reclamaciones posteriores, y en particular la reiteración de la misma solicitud a la clínica, no puede servir para computar un nuevo plazo» ${ }^{15}$. En el fallo que analizamos, la Corte de Apelaciones dio marcha atrás en su decisión, en cuanto acogió a tramitación el recurso presentado en

13. Sentencia del Séptimo Juzgado Civil de Santiago, rol V-53-2016, del 22 de agosto de 2016, que acoge por primera vez la rectificación de partida de un menor de edad amparado en la disconformidad entre el sexo asignado al nacimiento y la identidad de género. El juez que dictó dicha resolución fue objeto de una querella criminal por prevaricación judicial por parte de la ONG Comunidad y Justicia, la que fuera en definitiva desestimada por ser los hechos no constitutivos de delito. Véase causa RIT 6315-2017, del Séptimo Juzgado de Garantía de Santiago.

14. La primera de las sentencias señala: «2) Que en su presentación el recurrente señala haber tomado conocimiento del acto que lo motiva para deducir esta acción constitucional en enero de este año, circunstancia que se corrobora con la copia de la carta que se lee $[\ldots]$; 3) que habiendo deducido el presente recurso con fecha 15 de marzo de 2016, no puede ser acogido a tramitación por extemporáneo». Sentencia de la Corte de Apelaciones de Santiago, rol 20.170-2016, 16 de marzo de 2016.

15. En este sentido, señaló la Corte de Apelaciones de Santiago: «3) Que, tenido a la vista el Ingreso Corte número 20.170-2016, se advierte que los padres de T.I.A.F. han solicitado a Clínica Alemana la modificación de los datos de su hijo en varias oportunidades, como se advierte de la carta [...] fechada en enero de 2016, de modo que es posible colegir que los recurrentes han tomado conocimiento del acto que los motiva a recurrir de protección con una antelación superior al plazo previsto para su interposición, razón por la cual no puede ser acogido a tramitación por extemporáneo». Sentencia de la Corte de Apelaciones de Santiago, rol 32.429-2016, 28 de abril de 2016. 
los mismos términos y amparado en la misma negativa que días antes había declarado inadmisible.

La reclamación de extemporaneidad no fue tomada en cuenta, pues, a juicio de la Corte de Apelaciones, la acción, «que constituye el fundamento del presente recurso, continúa produciéndose $»^{16}$. De esta forma, se establece que una persona transgénero ve vulnerados sus derechos cada vez que no es llamada por su nombre social al momento de atenderse. El voto de minoría del fallo de la Corte Suprema postulaba rechazar la protección y revocar la sentencia por extemporaneidad.

\section{Apelación a la dignidad humana}

A falta de norma específica que resuelva el asunto por tratarse de un centro privado, y amparándose en la inexcusabilidad, la Corte de Apelaciones acudió a la dignidad de la persona, al advertir:

Que el derecho al completo y total desarrollo de la personalidad va de la mano con un concepto primordial que constituye la base de todos los principios y derechos que la Carta Fundamental y los tratados internacionales ratificados por nuestro país sobre la materia consagran, cual es el de la dignidad de la persona ${ }^{17}$.

\section{Vulneración del derecho fundamental de igualdad ante la ley}

La Constitución, en su artículo 19 número 2, asegura a todas las personas la igualdad ante la ley. De acuerdo con la Corte de Apelaciones, la igualdad debe entenderse como un principio y como un derecho que demanda ciertos enfoques, reivindicaciones y contenidos sustantivos. Citando a la Corte Internacional de Derechos Humanos, señala que la noción de igualdad:

Es inseparable de la dignidad esencial de la persona, frente a la cual es incompatible toda situación que, por considerar superior a un determinado grupo, conduzca a tratarlo con privilegio, o que, a la inversa, por considerarlo inferior, lo trate con hostilidad o de cualquier forma lo discrimine del goce de derechos que sí se reconocen a quienes no se consideran incursos en tal situación de inferioridad. En consecuencia, los Estados sólo pueden establecer distinciones objetivas y razonables cuando éstas se realicen con el debido respeto a los derechos humanos y de conformidad con el principio de la aplicación de la norma que mejor proteja los derechos de la persona ${ }^{18}$.

16. Sentencia de la Corte de Apelaciones de Santiago, rol 35.639-2016, 6 de octubre de 2016, considerando sexto.

17. Sentencia de la Corte de Apelaciones de Santiago, rol 35.639-2016, considerando décimo.

18. Sentencia de la Corte de Apelaciones de Santiago, rol 35.639-2016, considerando undécimo. La Corte hizo propia la Opinión Consultiva de la Corte Interamericana de Derechos Humanos OC-4/84, 
De acuerdo con la Corte de Apelaciones, la igualdad se afectó en virtud de no haberse respetado el principio de interés superior del niño (ISN). Por remisión al artículo 5 de la Constitución, los tratados internacionales ratificados por Chile que se encuentren vigentes resultan aplicables internamente. A partir del artículo 3 de la Convención sobre los Derechos del Niño y el Comité de los Derechos del Niño, la Corte de Apelaciones acogió la interpretación del ISN como un concepto triple. Primeramente, es un derecho sustantivo (de aplicación inmediata y reclamable ante los tribunales), luego un principio jurídico interpretativo, por lo que se elige la interpretación que satisfaga de manera más efectiva el ISN. Y, finalmente, se trata de una norma de procedimiento, esto es, el proceso de adopción de decisiones deberá incluir una estimación de las posibles repercusiones positivas o negativas de la decisión en el niño o los niños interesados. ${ }^{19}$ De esta manera, y a falta de norma, el ISN debe ser siempre una máxima de interpretación para los jueces perfectamente exigible en el ámbito de las relaciones privadas, como sería en la atención de salud.

\section{No modifica la interpretación anterior la existencia} de un procedimiento paralelo de rectificación de partida de nacimiento

La Corte de Apelaciones sostuvo que:

La actuación de la recurrida en orden a no incorporar en sus diversos registros al niño con su nueva identidad, establecida por sentencia ejecutoriada, sólo porque no se ha materializado la inscripción correspondiente en el Servicio de Registro Civil e Identificación, resulta completamente infundada, especialmente si se tiene en cuenta que la referida inscripción sólo tiene un sentido de publicidad, la que en caso alguno puede prevalecer sobre la dignidad de la persona, el interés superior del niño y la garantía constitucional de la igualdad; derechos todos que se han conculcado por la recurrida con su negativa caprichosa y antojadiza que sin duda la torna arbitraria, por lo que la acción de protección será acogida ${ }^{20}$.

\section{Aceptación del recurso y condena en costas}

Fue por ello que la Corte de Apelaciones acogió el recurso, «debiendo en consecuencia Clínica Alemana de Santiago inscribir en la correspondiente ficha clínica de la menor su identidad legal XX, como asimismo en todo ámbito de actuación de la recurrida para con la niña $»^{21}$. La recurrida fue expresamente condenada en costas.

\footnotetext{
«Propuesta de modificación a la Constitución Política de Costa Rica relacionada con la naturalización», del 19 de enero de 1984.

19. Sentencia de la Corte de Apelaciones de Santiago, rol 35.639-2016, considerando duodécimo.

20. Sentencia de la Corte de Apelaciones de Santiago, rol 35.639-2016, considerando duodécimo.

21. Sentencia de la Corte de Apelaciones de Santiago, rol 35.639-2016, parte resolutiva.
} 


\section{Conclusión}

Si bien en materia de atención pública existe una norma expresa que obliga a tratar a las personas transgénero por su nombre social, a la misma conclusión se puede llegar respecto de pacientes que se atienden en el sector privado mediante una interpretación armónica de nuestro ordenamiento, en especial en virtud de la Convención de Naciones Unidas Sobre los Derechos del Niño, de la Ley 20.584 (que consagra el principio de autonomía y dignidad que subyace a la ficha clínica) y el Reglamento 41.

Enfrentada al escenario de ausencia normativa, la Corte de Apelaciones, en fallo ratificado por la Corte Suprema, ha establecido el derecho a ser tratado por el nombre social en materias vinculadas a la atención de salud, respecto de un menor de edad, de acuerdo con la dignidad de la persona, igualdad ante la ley y el ISN. Dicho fallo, además de la interpretación de las normas sobre derechos de los pacientes ya expuesta, invita a incorporar en los centros privados, al menos como buenas prácticas, los protocolos que rigen a las instituciones públicas. De esta manera, no tendremos tratos discriminatorios entre pacientes y se respetará el derecho al nombre y al trato digno del paciente, en todo momento y circunstancia, como mandata la Ley 20.584 .

\section{Referencias}

Fernández Hierro, José Manuel (2002). La historia clínica. Granada: Comares.

Ghersi, Carlos y Celia Weingarten (2005). Historia clínica. Rosario: Editorial Jurídica Nova Tesis.

Noriega Potocnjak, Fernando (2011). La negligencia médica ante la doctrina y jurisprudencia nacionales. Santiago: Ediciones Jurídicas de Santiago.

Rebollo Delgado, Lucrecio y Yolanda Gómez Sánchez (2008). Biomedicina y protección de datos. Madrid: Dykinson.

Ruiz Orejuela, Wilson (2004). Responsabilidad médica estatal. Medellín: Librería Jurídica Sánchez R. Limitada.

Terribas, Nuria (2012). «Derechos del paciente». En Juan Pablo Becerra y Carmen Astete (editores), Bioética clínica (pp. 178-204). Santiago: Mediterráneo.

\section{Sobre el autor}

Pablo Eterovic Barreda es licenciado en Ciencias Jurídicas y Sociales por la Facultad de Derecho de la Universidad de Chile. Estudiante del Magíster en Derecho Tributario en la misma casa de estudios. Su correo electrónico es pabloeterovicb@ gmail.com. 